\title{
Zwischen Aushandlung, Normierung und Moralisierung
}

\author{
Eine Einführung in den Themenschwerpunkt
}

Sozialpädagogisches Handeln ist normativ. Auch wenn diese Erkenntnis nicht neu ist, wird Professionalität in der Sozialen Arbeit zumeist jenseits sozialer Normen und emotionaler Verstrickungen der handelnden Professionellen diskutiert oder Normen werden als Bedrohungen für professionelles Agieren thematisiert. Werden diese hingegen als konstitutiver Bestandteil für das Agieren sozialpädagogischer Fachkräfte anerkannt, könnten jenseits vorschneller Defizitdiagnosen Überlegungen angestellt werden, wie der Umgang mit sozialen Normen als Teil einer reflexiven Professionalität gelingen könnte.

$\mathrm{I}$ m Sprechen über sozialpädagogische Professionalität wird zumeist das reflexive, geplante und eigenständige, nachvollziehbare, möglichst unterstützende und die Autonomie von Adressat_innen wahrende, solidarische, aber ansonsten emotional besonnene und normativ enthaltsame Agieren betont. Wenn Emotionen eine Bedeutung zugesprochen wird, dann wird Empa-

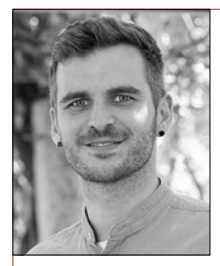

Julian Sehmer

Hochschule für angewandte Wissenschaft und Kunst (HAWK), Holzminden, Deutschland

*1990; Verwaltungsprofessur für Soziale Arbeit an der HAWK Holzminden und Sprecher des Instituts für Theorie und Empirie des Sozialen (ITES).

Julian.sehmer@ites-werkstatt.de

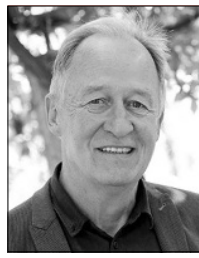

\section{Werner Thole}

TU Dortmund, Dortmund, Deutschland

*1955; Prof. Dr. phil, TU Dortmund, bis April 2021 Hochschullehrer für Erziehungswissenschaft mit dem Schwerpunkt Soziale Arbeit und außerschulische Bildung an der Universität Kassel. wthole@uni-kassel.de

Zusammenfassung Soziale Normen als spezifische Annahmen von Normalität bestimmen nicht nur, wie Subjekte über sich selbst und andere nachdenken. Sie sind auch Ausgangspunkt jeder Bezugnahme auf Andere. Der Beitrag entfaltet ein Verständnis von Normen anhand der These, dass soziale Normen sowohl konstitutiver Bestandteil Sozialer Arbeit sind als auch einen zu problematisierenden Gegenstand eines reflexiven Umgangs mit Professionalität darstellen.

Schlüsselwörter Soziale Normen, Professionalität, Normalität, Normalisierung, Moralisierung, Subjektivierung, Ethische Gewalt thie als Voraussetzung für eine förderliche Beziehung zu den Adressat_innen herausgestellt. Sozialpädagogische Profis sollen möglichst ungeachtet von Sympathien oder Antipathien fallbezogen und fallübergreifend Arbeitsbündnisse initiieren und dabei gekonnt das richtige Verhältnis von Nähe und Distanz austarieren (Dörr und Müller 2019). Auch wenn Fachkräfte Kontrollaufgaben wahrnehmen, sollen sie dies wertschätzend, transparent und auf Basis gesellschaftlicher Mandatierungen und professionsethischer Standards tun und dabei dennoch den teils widersprüchlichen Erwartungen gerecht werden (Thieme 2017; Thole et al. 2018).

Angesichts dieser Komplexität werden soziale Normen und persönliche emotionale Verstrickungen überwiegend dann thematisiert, wenn sie als problematisch markiert werden. Dies beispielsweise weil Deutungen und Rhetoriken aufscheinen, die dem Spektrum gruppenbezogener Menschenfeindlichkeit (Decker und Brähler 2020; Heitmeyer 2018) zugeordnet werden können und sozialpädagogische Unterstützung für vulnerable Gruppen in Frage stellen (Gille et al. 2021). Solche Deutungen und darauf basierendes Handeln verhindern, dass sozialpädagogisch verantwortete Räume zu Orten werden, an denen sich auch jene Gruppen sicher fühlen können, die aufgrund ihrer Differenz zu hegemonialen Normvorstellungen besonders häufig zum Ziel von Anfeindungen, Ausgrenzungen oder Othering werden (Riegel 2017). Dabei sind soziale Normen und deren Aushandlung konstitutiv für Soziale Arbeit und entsprechend auch dann präsent, wenn sie nicht in Form "problematischer Ablehnungskonstruktionen“ (Thole 2020) aufscheinen. 


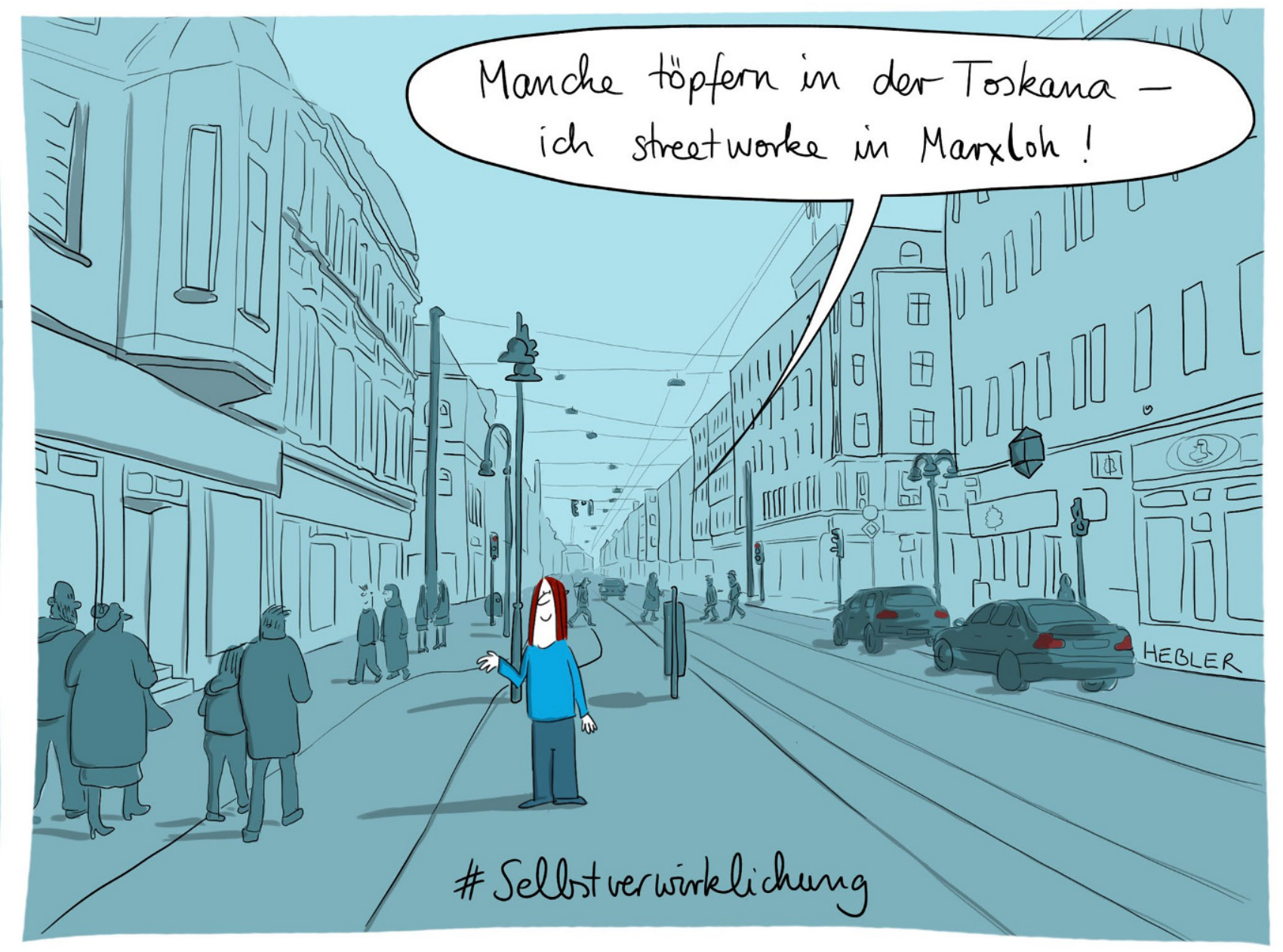

Während professionstheoretische Betrachtungen die Bedeutung wissenschaftlicher und systematisch gewonnener methodischer Wissensbestände hervorheben, kommen Studien, die diese gezielt im Feld zu entdecken versuchen, eher zu ernüchternden Ergebnissen (Thole et al. 2016). Wird den entsprechenden Befunden gefolgt, markieren Fachkräfte in ganz unterschiedlichen Handlungsfeldern insbesondere lebensweltliche Wissensbestände und biographische Erfahrungen als bedeutsam für ihr eigenes Handeln (Kaul 2019; Retkowski et al. 2011). Ließe sich hier aus professionstheoretischer Sicht ein Defizit sozialpädagogischer Professionalität konstatieren, erinnert dieser Befund vor allem daran, dass sich die Professionalisierung sozialpädagogischen Handelns weder technologisch lösen lässt noch über einen einfachen Transfer wissenschaftlicher Erkenntnisse in die Handlungspraxis der Sozialen Arbeit bewerkstelligt werden kann (Sehmer et al. 2019). Stellt sich Soziale Arbeit vor allem als kommunikative Arbeit mit Adressat_innen dar, die deren Le- bensformen zum Gegenstand macht (Thole und Ziegler 2018), erfordert sie auch die Transformation von nüchternen Informationen in lebendiges Wissen und insofern auch deren Aufladung mit Emotionen und Einbettung in - von Vorstellungen vom „richtigen “ und „normalen “ Leben getragene - Selbst- und Weltdeutungen. Die Befunde verweisen darauf, davon auszugehen, dass das darin eingelagerte wissenschaftliche Wissen nur dann sedimentiert werden kann, wenn es Anschluss an die Deutungen der Professionellen darüber findet, wie sie sich selbst und die Welt verstehen.

In den letzten Jahrzehnten hat die zunehmende Verschränkung von persönlichen, emotionalen sowie von normativen Bezügen und beruflichem Handeln nicht nur in der Sozialen Arbeit zusätzliche Dynamisierungen erfahren. Teil gesellschaftlicher Entwicklungen der Spätmoderne, so legen aktuelle Gesellschaftsanalysen (Reckwitz 2019) nahe, ist es auch, dass von beruflich Engagierten zunehmend erwartet wird, nicht mehr bloß „Dienst nach Vorschrift“ zu machen, sondern sich mit 


\section{Extrablick: Normen in der Sozialen Arbeit}

ihrer ganzen Persönlichkeit einzubringen und in ihrem beruflichen Alltag Selbstverwirklichung zu suchen. Damit tritt die Orientierung auf reine Erwerbsarbeit und Einkommen zunehmend in den Hintergrund, während die Grenzen zwischen Beruf und privatem Alltagsleben brüchig werden und Fachkräfte auch im beruflichen Handeln verstärkt Anerkennung, positive Bestärkung, Zufriedenheit und Glück suchen. Teil dieser Entwicklung, die Andreas Reckwitz (2019) als „Singularisierung " beschreibt, ist die Zunahme an Bewertungen von Objekten, Orten, Ereignissen und eben auch Subjekten unter anderem anhand neuer ethischer und ästhetischer Kriterien. Für diese - singularisierten - Orientierungen werden ethisch-normative Bewertungen bedeutsam, die teilweise auch quer zu den bisherigen normativen $\mathrm{Pa}$ radigmen der Sozialen Arbeit wie Solidarität, Gerechtigkeit und Gleichheit zu liegen scheinen. Für Fachkräfte könnte bedeutsamer werden, dass ihre Suche nach Selbstwirksamkeit und Authentizität, Eigenständigkeit und Kreativität ihnen auch von Adressat_innen gespiegelt wird. Entsprechend spielen neben den von Professionstheorien hervorgehobenen Aspekten (neue) Subjektivierungspraktiken auch in Bezug auf die Gestaltung von Beziehungen zwischen den Fachkräften und den jeweils gegebenen Spannungen und Konflikten eine wesentliche Rolle in der Praxis Sozialer Arbeit.

\section{Soziale Normen zwischen produktiver Kraft und ethischer Gewalt}

Soziale Normen bezeichnen Vorstellungen davon, wie etwas ist und wie es sein soll. Sie bestimmen, wie die Einzelnen über sich nachdenken können und wie sie die Welt, ihre Beziehungen und vielleicht auch sich selbst verstehen und wahrnehmen und werden anhand von Interaktionen mit anderen von der Kindheit an geformt und verinnerlicht (Butler 2014). Anders als Werte können sie von einzelnen Subjekten nur schwer expliziert werden, weil sie in die eigenen Selbstbilder über alle erlebten Begegnungen und Beziehungen mit anderen eingeflochten sind. „Sozial“ sind Normen also in dem Sinne, dass sie einerseits gesellschaftlich präformiert sind und zugleich ausgehandelt und über die Interaktion mit anderen angeeignet, transformiert und reproduziert werden. Dabei gibt es keine exklusiven Räume der Aushandlung. Diese erfolgt in alltäglichen Interaktionen, sodass in jede Bezugnahme auf Einzelne oder Gruppen soziale Normen als spezifische Vorstellungen von Normalität und Legitimität eingebettet sind und Erwartungen, diesen zu entsprechen, an sie vermittelt werden.

Über die Adressierung von und mit Normen beginnen Menschen, sich selbst als Subjekte zu begreifen und in einer bestimmten Art und Weise andere, sich selbst und die Welt anhand dieser verinnerlichten Normalitätsvorstellungen abzugleichen - mit anderen Worten: „Das ,Ich“ hat gar keine Geschichte von sich selbst, die nicht zugleich die Geschichte seiner Beziehung - oder seiner Beziehungen - zu bestimmten Normen ist" (Butler 2014, S. 15). Soziale Normen sind also immer auch eng mit Machtverhältnissen verbunden, bilden soziale Ordnungen - etwa in Bezug auf Geschlechter, Klassen und Körper - ab und bringen diese hervor. Sie sind produktive Kraft, indem sie Subjekte hervorbringen, setzen aber auch Grenzen dafür, wer oder was noch als normal und legitim gelten kann und produzieren Leid für diejenigen, die ihnen nicht entsprechen. So wird etwa anhand des Beitrags von Davina Höblich und Hannah Goede in diesem Heft deutlich, wie die Vorstellung von Heteronormativität dazu führt, dass sich einzelne erst als LSBTI*Q-Personen denken und erfahren können, diesen aber auch zumutet, im Alltag an Grenzen zu stoßen, die sich aus ihrer zugeschriebenen Differenz zur hegemonialen Vorstellung von Normalität ergeben (Eribon 2017, 2019; Krell und Oldemeier 2017). Schließlich ist auch das gesellschaftliche Erinnern bestimmter Ereignisse eng mit sozialen Normen, Macht und Anerkennung verbunden, sodass manche Ereignisse aus dem sozialen Gedächtnis verschwinden oder erst wieder erinnerbar werden, wenn sich soziale Normen verändern (Fischer 2021).

Auch im Kontext von Armutsdeutungen lassen sich bestimmte soziale Normen rekonstruieren, wie es Anja Kerle in ihrem Beitrag unternimmt. Hier weisen verschiedene Studien darauf hin, dass sozialpädagogische Fachkräfte Armut nicht durchgängig als gesellschaftliche Problematik fassen, sondern über Individualisierung in ein persönlich verschuldetes Unvermögen einzelner Erwachsener transformieren, dem dann pädagogisch begegnet werden kann (Simon et al. 2019). In solchen Fällen, in denen Einzelne mit der Verletzung einer Norm konfrontiert werden - in diesem Fall so zu leben, dass die eigenen Kinder nicht von Armut betroffen sind -, der sie etwa aufgrund sozialer Ungleichheiten gar nicht entsprechen können, spricht Judith Butler (2014) von „ethischer Gewalt“. Ethische Gewalt geht von den genannten heteronormativen Vorstellungen, aber auch von rassistischen, klassistischen, ableistischen Deutungen aus.

\section{Aushandlungen von Normalität}

Auch wenn soziale Normen Quelle ethischer Gewalt sein können, können Fachkräfte der Sozialen Arbeit nicht auf normative Bezüge verzichten. Da soziale Normen als Vorstellungen von Normalität auch bestimmen, wie Subjekte sich und andere denken - sie in diesem 
Sinne subjektivieren - sind sie Ausgangspunkt jeder Bezugnahme auf andere und auch für Soziale Arbeit zentral. So führen etwa Fabian Kessl und Hans-Uwe Otto (2012, S. 1306) aus, dass Soziale Arbeit „organisierte Prozesse einer aktiven Unterstützung und geplanten Beeinflussung von Subjektivierungsweisen " beschreibt, „die als sozial problematisch markiert werden. Subjektivierung wird dabei als Prozess ambivalenter Gleichzeitigkeit von Unterwerfung und Subjektwerdung verstanden. " Erst vor dem Hintergrund sozialer Normen werden diejenigen, die ihnen nicht entsprechen, als differente Andere sicht- und adressierbar. Ihnen kann dann Hilfe und Unterstützung ebenso angeboten werden, wie im Zweifelsfall auch Kontrolle und Kriseninterventionen als notwendig angesehen werden können. Adressat_ innen der Sozialen Arbeit werden also auch zu Adressat_innen, weil sie anhand von Normen als ,anders“ oder „problematisch“ erscheinen (Plößer 2014).

Zwar ist in den Handlungsfeldern Sozialer Arbeit ein Bewusstsein dafür beobachtbar, dass das Sprechen von „Normalität“ nicht unproblematisch ist, gleichwohl ist gerade die (Wieder-)Herstellung von Normalität vielfach Ziel sozialpädagogischer Interventionen. Wenn etwa, wie Mischa Engelbracht in diesem Schwerpunkt ausführt, in Einrichtungen der stationären Kinder- und Jugendhilfe ein komplexes System aus Regeln, Anreizen und Strafen konstruiert wird, um als problematisch angesehenes Verhalten und Orientierungen von Kindern und Jugendlichen so weit zu korrigieren, dass ihr Handeln nicht mehr von den Fachkräften als abweichend gedeutet wird. Oder wenn, wie Holger Schmidt zeigt, Adressat_innen und Fachkräfte die Normalität innerhalb der Offenen Kinder- und Jugendarbeit in einer Arbeitsbeziehung aushandeln und dabei ganz unterschiedliche soziale Normen aufeinandertreffen können.

Wenn es so ist, dass es vor allem biographische Bezüge sind, die von den Fachkräften als Begründung für ihre Entscheidungen angeführt werden, so sind es vor allem subjektiv angeeignete und teilweise milieuspezifische Normvorstellungen, die über die Fachkräfte an die Adressat_innen gerichtet werden. Die Herausforderung besteht für die beruflichen Akteur_innen der Sozialen Arbeit folglich darin, mit den Annahmen von Normalität vorsichtig zu hantieren und nicht die eigene Normalität unreflektiert als Standard zu setzen und als gegebene, nicht zu hinterfragende Normalität zu kommunizieren - Normalität also als breites Kontinuum zu reflektieren, innerhalb dessen unterschiedliche Lebensformen ihren Raum finden können. Gelingt dies nicht, besteht die Gefahr der Ausübung ethischer Gewalt, weil die Fachkräfte auch davon absehen, Normalität im Plural zu definieren und die jeweils gegebenen gesellschaft- lichen Bedingungen zu identifizieren, die zu sozialen Ungleichheiten und differenten Gestaltungen des Lebens führen. Oder weil die Normalität von Adressat_innen ohne Mandat als illegitim erkannt und verändert werden soll, Sozialpädagog_innen also ihre Macht derart überstrapazieren, wie es vielfach unter dem Begriff der „Kolonialisierung von Lebenswelten“ (Rauschenbach und Gängler 1984) beschrieben worden ist. Aus empirischer Perspektive werden immer wieder Praktiken von Kindern und Jugendlichen mit dem Ziel der Normalisierung danach angefragt, ob sie nicht Wege zur Mündigkeit blockieren und stattdessen Prozesse der $\mathrm{Zu}$ richtung, Züchtigung oder „Dressur“ befördern (vgl. u. a. Degener et al. 2020). Empirische Impulse können so dazu beitragen, die Normalitätsvorstellungen von Fachkräften kritisch zu hinterfragen und Normalität als auch anders möglich sichtbar werden zu lassen.

Dass Vorstellungen und Orientierungen von Normalität für die Soziale Arbeit dennoch bedeutsam sind, lässt sich beispielsweise am sozialpädagogisch verantworteten Kinderschutz erkennen. Der hier zentrale Begriff der Kindeswohlgefährdung entzieht sich der rechtlichen Bestimmung, weil er ein normatives Konstrukt ist, das nur zeit-, kontext- und kulturspezifisch zu verstehen ist und einer feinfühligen, dialogischen und fallspezifischen Diagnostik bedarf. Hier gilt es die unscharfe Trennlinie zwischen einer Erziehung, die einer anderen, vielleicht nicht durchgängig als förderlich angesehenen Normalität folgt, und einer, die unbedingt zu vermeidenden Schaden und Leid bei Kindern und Jugendlichen verursachen könnte, stets neu zu identifizieren. Um dies zu erkennen, hantieren die Fachkräfte auch mit spezifischen Vorstellungen und Konzepten von Kindern und Kindheiten (vgl. u. a. Simon 2018; Sehmer 2018). Befunde ${ }^{1}$ zum Umgang mit Normen im Kinderschutz deuten an, dass sich Fachkräfte als Vertreter_innen einer aus ihrer Sicht allgemeingültigen und vermeintlich gesellschaftlich konsensualen Normalität inszenieren, der sich auch die Adressat_innen unterwerfen sollen. Über diese Inszenierung, mit der sich die sozialpädagogischen Akteur_innen selbst als Sprecher_innen einer hegemonialen Perspektive in Stellung bringen, sichern sie sich eine gute Ausgangslage, um im vermeintlichen Interesse der Kinder und Jugendlichen Maßnahmen durchzusetzen. Zugleich werden jedoch potenziell mögliche Aushandlungsprozesse blockiert. Insbesondere werden Normen in Bezug auf Geschlechter- und Generationenverhältnisse reproduziert, die sich an einem spezifischen Bild der heterosexuellen Kernfamilie orientieren. Demnach kommt besonders den Müttern die Verantwortung zu, das gute Aufwachsen ihrer Kinder zu sichern, während jede Beteiligung von Vätern an der Erziehung eher als 


\section{Extrablick: Normen in der Sozialen Arbeit}

freiwilliges Engagement honoriert wird. Fehlendes Engagement von Vätern wird in den untersuchten Fällen hingegen nur dann kritisch angefragt, wenn es auch mit mangelnder finanzieller Unterstützung einhergeht.

Das über Normen konstruierte mutterzentrierte Familienbild der Fachkräfte wird im Kinderschutz also organisational in ein Hilfesystem übersetzt, das primär auf das Agieren der Mütter ausgerichtet ist und erst subsidiär auch Väter und weitere Personen des sozialen Netzwerks einbezieht. So werden zumeist nur Mütter zu Terminen eingeladen, nur ihre Perspektiven dokumentiert und primär sie werden für auftretende Schwierigkeiten verantwortlich gemacht. Den Müttern wird aufgrund allgemeiner, als gesellschaftlich hegemonial markierter Normen und weniger über ihre jeweilige Person eine höhere Bedeutung für das Aufwachsen ihrer Kinder zugeschrieben, die mit höherer Beteiligung an Entscheidungsprozessen einhergeht. Zugleich sehen sie sich im Falle von biografischen Verläufen der Heranwachsenden, die aus Sicht der Fachkräfte nicht optimal sind, auch hauptsächlich mit möglichen Schuldzuschreibungen konfrontiert.

Im Fall von gebrochenen Wegen durch die Kindheit und Jugend oder von als ungenügend eingeschätzten Versorgungen von Kindern und Jugendlichen kann dies, vor allem auch im Kontext von Armutslagen, zu moralisierenden Zuschreibungen führen. Der Verstoß gegen die als allgemeingültig präsentierten sozialen Normen kann durch die Fachkräfte dann als mangelnde Empathie, Liebe oder Sorge der Mütter zu ihren Kindern gedeutet werden, während alternative Erklärungen für das Agieren überwiegend nicht mehr in Betracht gezogen werden (vgl. Marks und Sehmer 2017).

Verdeutlichen sollen diese exemplarischen Sondierungen zur Bedeutung sozialer Normen in der Sozialen Arbeit, dass diese für die sozialpädagogische Praxis nicht nur am Rande bedeutsam sind, sondern diese konstitutiv und durchgehend mitbestimmen. Dabei können sie sowohl Gegenstand von Aushandlungen sein wie auch implizit die Deutungen der Fachkräfte von und den Umgang mit Adressat_innen rahmen.

\section{Zwischen Aushandlung und Dekonstruktion}

Sozialpädagogische Fachkräfte können nicht abgekoppelt von sozialen Normen und darüber fundierten Normalisierungsintentionen agieren. Gleichwohl sind sie gefordert, die eigenen Deutungen von Normalität kritisch anzufragen, um die Reproduktion ethischer Gewalt über Angebote Sozialer Arbeit zu verhindern. Wenn dies gelingen soll, müssen soziale Normen zum Gegenstand reflexiver Dekonstruktionen ebenso werden wie zugleich auch eine Aushandlung von Normalität zwi- schen Fachkräften und Adressat_innen unter Einbezug wissenschaftlichen Wissens und unter Berücksichtigung gesellschaftlicher Erwartungshaltungen erfolgen sollte. Dies fordert professionstheoretische Zugänge, normative Bezüge ebenso wie emotionale Verstrickungen in der sozialpädagogischen Praxis nicht vorschnell als defizitär abzuqualifizieren, sondern als konstitutives Element einer immer auch von Widersprüchen, Antinomien und Paradoxien durchwebten sozialpädagogischen Praxis zu verstehen.

Eingegangen. 30. März 2021

Angenommen. 30. März 2021

Funding. Open Access funding enabled and organized by Projekt DEAL.

Open Access. Dieser Artikel wird unter der Creative Commons Namensnennung 4.0 International Lizenz veröffentlicht, welche die Nutzung, Vervielfältigung, Bearbeitung, Verbreitung und Wiedergabe in jeglichem Medium und Format erlaubt, sofern Sie den/die ursprünglichen Autor(en) und die Quelle ordnungsgemäß nennen, einen Link zur Creative Commons Lizenz beifügen und angeben, ob Änderungen vorgenommen wurden.

Die in diesem Artikel enthaltenen Bilder und sonstiges Drittmaterial unterliegen ebenfalls der genannten Creative Commons Lizenz, sofern sich aus der Abbildungslegende nichts anderes ergibt. Sofern das betreffende Material nicht unter der genannten Creative Commons Lizenz steht und die betreffende Handlung nicht nach gesetzlichen Vorschriften erlaubt ist, ist für die oben aufgeführten Weiterverwendungen des Materials die Einwilligung des jeweiligen Rechteinhabers einzuholen.

Weitere Details zur Lizenz entnehmen Sie bitte der Lizenzinformation auf http://creativecommons.org/licenses/by/4.0/deed.de.

1. Die Ergebnisse wurden im Rahmen des aktuell laufenden Dissertationsprojektes „Sozialpädagogische Subjekt- und Adressierungspraktiken. Konturierungen der normativen Rabmungen sozialpädagogischer Praxis" von Julian Sehmer erarbeitet.

\section{Literatur}

Butler, J. (2014). Kritik der ethischen Gewalt. Adorno-Vorlesungen 2002. Frankfurt am Main: Suhrkamp.

Decker, O., \& Brähler, E. (2020). Autoritäre Dynamiken. Alte Ressentiments - neue Radikalität. In E. Brähler \& O. Decker (Hrsg.), Autoritäre Dynamiken. Neue Radikalität - alte Ressentiments. Leipziger Autoritarismus Studie 2020(S. 15-26). Gießen: Psychosozial-Verlag.

Degener, L., Kunstreich, T., Lutz, T., Mielich, S., Muhl, F., Rosenkötter, W., \& Schwagereck, J. (2020). Dressur zur Mündigkeit? Über die Verletzung von Kinderrechten in der Heimerziehung. Weinheim: Beltz Juventa.

Dörr, M., \& Müller, B. (Hrsg.). (2019). Nähe und Distanz. Ein Spannungsfeld pädagogischer Professionalität (4. Aufl.). Weinheim: Beltz Juventa.

Eribon, D. (2017). Gesellschaft als Urteil. Klassen, Identitäten, Wege. Berlin: Suhrkamp.

Eribon, D. (2019). Betrachtungen zur Schwulenfrage. Berlin: Suhrkamp. 
Fischer, G. (2021). Wenn erinnern stört - Wie ein rechtsextremer Mord in Baden-Württemberg aus dem hegemonialen Diskurs verschwindet. In J. Sehmer, S. Simon, J. Ten Elsen \& F. Thiele (Hrsg.), recht extrem? Dynamiken in zivilgesellschaftlichen Räumen. Wiesbaden: Springer VS. im Erscheinen.

Gille, C., Jagusch, B., Krüger, C., \& Wéber, J. (2021). Ambivalente Verhältnisse und steigende Einflussnahmen: Soziale Arbeit und die extreme Rechte. In J. Sehmer, S. Simon, J. Ten Elsen \& F. Thiele (Hrsg.), recht extrem? Dynamiken in zivilgesellschaftlichen Räumen. Wiesbaden: Springer VS. im Erscheinen.

Heitmeyer, W. (2018). Autoritäre Versuchungen. Signaturen der Bedrobung 1. Berlin: Suhrkamp.

Kaul, I. (2019). Bildungskonzepte von Pädagoginnen in Kindertageseinrichtungen. Eine empirisch-rekonstruktive Untersuchung biografischer Wege. Wiesbaden: Springer VS.

Kessl, F., \& Otto, H.-U. (2012). Soziale Arbeit. In G. Albrecht \& A. Groenemeyer (Hrsg.), Handbuch soziale Probleme (S. 1306-1331). Wiesbaden: VS.

Krell, C., \& Oldemeier, K. (2017). Coming-out - und dann...?! Comingout-Verläufe und Diskriminierungserfahrungen von lesbischen, schwulen, bisexuellen, trans* und queeren Jugendlichen und jungen Erwachsenen in Deutschland. Opladen: Barbara Budrich.

Marks, S., \& Sehmer, J. (2017). Familiale Autonomie im Kinderschutz. Sozialer Sinn, 18(2), 203-229.

Plößer, M. (2014). Normen, Subjekte, Soziale Arbeit. Queere Perspektiven auf ein ambivalentes Verhältnis. Sozialmagazin: die Zeitschrift für Soziale Arbeit, 39(3/4), 14-20.

Rauschenbach, T., \& Gängler, H. (1984). Halbierte Verständigung - Sozialpädagogik zwischen Kolonialisierung und Mediatisierung lebensweltlichen Eigensinns. In S. Müller (Hrsg.), Verstehen oder kolonialisieren? Grundprobleme sozialpädagogischen Handelns und Forschens (S. 145168). Bielefeld: Kleine.

Reckwitz, A. (2019). Die Gesellschaft der Singularitäten. Zum Strukturwandel der Moderne. Berlin: Suhrkamp.

Retkowski, A., Schäuble, B., \& Thole, W. (2011). „Diese Familie braucht mehr Druck ...". Praxismuster im Allgemeinen Sozialen Dienst - Rekonstruktion der Bearbeitung eines Kinderschutzfalles. Neue Praxis: Zeitschrift für Sozialarbeit, Sozialpädagogik und Sozialpolitik, 41(5), 485-504.

Riegel, C. (2017). Queere Familien in pädagogischen Kontexten. Zwischen Ignoranz und Othering. Opladen: Barbara Budrich.

Sehmer, J. (2018). Zur Konstruktion des Kindes durch Fachkräfte eines Allgemeinen Sozialen Dienstes. In I. Kaul, D. Schmidt \& W. Thole (Hrsg.) Kinder und Kindheiten (S. 131-151). Wiesbaden: Springer VS.

Sehmer, J., Marks, S., \& Thole, W. (2019). Wissen im Dialog. Sozial Extra, 43(4), 259-262.

Simon, S. (2018). Belastete Kindheit - belastete Kinder? In I. Kaul, D. Schmidt \& W. Thole (Hrsg.), Kinder und Kindheiten (S. 13-32). Wiesbaden: Springer.

Simon, S., Prigge, J., Lochner, B., \& Thole, W. (2019). Deutungen von Armut - Pädagogische Thematisierungen von und Umgangsweisen mit sozialer Ungleichheit in Kindertageseinrichtungen. Neue Praxis: Zeitschrift für Sozialarbeit, Sozialpädagogik und Sozialpolitik, 19(5), 395-415.

Thieme, N. (2017). Hilfe und Kontrolle. In F. Kessl, E. Kruse, S. Stövesand \& W. Thole (Hrsg.), Soziale Arbeit - Kernthemen und Problemfelder (S. 17-24). Opladen: Barbara Budrich.

Thole, W. (2020). Problematische Ablehnungskonstruktionen. Sozial Extra, 44(2), 107-112.

Thole, W., \& Ziegler, H. (2018). Soziale Arbeit als praktische Kritik der Lebensformen. Soziale Passagen, 10(1), 7-28.

Thole, W., Milbradt, B., Göbel, S., \& Rißmann, M. (2016). Wissen und Reflexion. Der Alltag in Kindertageseinrichtungen im Blick der Professionellen. Kasseler Edition soziale Arbeit, Bd. 4. Wiesbaden: Springer VS.

Thole, W., Klomann, V., Rätz, R., Wolff, R., Marks, S., Sehmer, J., Biesel, K., \& Messmer, H. (2018). Für einen unterstützungs-orientierten Kinderschutz. Kinderschutzpraxis zwischen Hilfe- und Kontrollauftrag, professionellen Einschätzungsprozessen und der Initiierung von Hilfs- und Unterstützungsangeboten. Sozial Extra, 42(4), 50-52.

Hier steht eine Anzeige. Springer 